\title{
Layering in sedimentation of suspensions of charged colloids: Simulation and theory
}

\author{
A. Cuetos, ${ }^{1, *}$ A.-P. Hynninen, ${ }^{1, \dagger}$ J. Zwanikken, ${ }^{2}$ R. van Roij, ${ }^{2}$ and M. Dijkstra ${ }^{1}$ \\ ${ }^{1}$ Soft Condensed Matter, Utrecht University, Princetonplein 5, 3584 CC Utrecht, The Netherlands \\ ${ }^{2}$ Institute for Theoretical Physics, Utrecht University, Leuvenlaan 4, 3584 CE Utrecht, The Netherlands
}

(Received 7 October 2005; revised manuscript received 15 March 2006; published 7 June 2006)

\begin{abstract}
We study the equilibrium sediment of a multicomponent system of charged colloids using primitive model Monte Carlo simulations, which include counterions explicitly. We find separation of the different colloidal components into almost pure layers, where colloids with large charge-to-mass ratio sediment higher in the sample. This effect appears due to a competition between ionic entropy, gravitational energy, and electrostatic energy. Our simulations provide a direct confirmation of recent theoretical predictions on the sedimentation of multicomponent mixtures of charged colloids in regimes with relatively low total densities and low colloidal charges. To explore the limitations of the theory we perform simulations at higher total densities for monodisperse and multicomponent systems and at stronger electrostatic couplings by increasing the colloidal charge for monodisperse suspensions. We find good agreement between theory and simulation when the colloidal charge is increased in the monodisperse case. However, we find deviations between simulations and theory upon increasing the total densities in the monodisperse and multicomponent systems. The density profiles obtained from simulations are more homogeneous than those predicted by theory. The spontaneous formation of layered structures predicted by the theory and found by simulation can serve as a useful tool to separate different components from a mixture of charged colloids.
\end{abstract}

DOI: 10.1103/PhysRevE.73.061402

PACS number(s): 82.70.Dd, 61.20.Ja, 05.20.Jj

\section{INTRODUCTION}

Sedimentation equilibrium of colloidal particles in a gravitational field seems, at first sight, a problem solved in the past. It is indeed true that this topic has been studied in detail for a long time. For example, Perrin [1] determined the Boltzmann constant $k_{B}$ (and from this Avogadro's number) by comparing the measured density profile $\rho(z)$ of a dilute system of particles with buoyant mass $m$ in a medium at temperature $T$ with the theoretically predicted barometric law $\rho(z) \propto \exp \left(-m g z / k_{B} T\right)$. Here $g$ is the gravitational acceleration, and $z$ is the height. This Boltzmann distribution is the result of a balance in a competition between minimum potential energy (favoring a large concentration of particles at the bottom) and maximum entropy (favoring a homogeneous distribution of particles). The barometric distribution is valid only for dilute dispersions, in which colloid-colloid interactions can be ignored. In more recent times, sedimentation equilibrium has received a renewed attention, as it was for instance realized that the hard-sphere bulk equation of state can be measured in a large density range from a single measurement of the equilibrium density profile of colloidal hard spheres in the Earth's gravity field [2-5]. The same procedure was also used to obtain the equation of state for a whole density range from the inversion of the sedimentation profile measured in a single Monte Carlo simulation of infinitely thin hard platelets $[6,7]$ and of hard rods [8]. Nontrivial sedimentation equilibria, including orientational inhomogeneities, layering effects, multiphase equilibria, and floating liquid phases, have been predicted recently in hard-sphere

\footnotetext{
*Electronic address: a.cuetos@phys.uu.nl

${ }^{\dagger}$ Present address: Department of Chemical Engineering, Princeton University, Princeton NJ 08544.
}

fluids [9], colloidal hard-rod dispersions [10,11], platelets [12], mixtures of platelets and nonadsorbing polymer [13], colloid-polymer mixtures $[14,15]$, and binary hard-sphere mixtures [16].

Very recently, quite some attention has been devoted to sedimentation equilibrium of charged colloids in an electrolyte solution with cations and anions, in particular under conditions of low salinity. Even at rather low colloid concentrations, large deviations from the barometric distribution have been theoretically predicted [5,17-19], experimentally observed [20-23], and simulated [18,24]. For a monodisperse system of colloids with a charge $Z e$ (with $e$ the proton charge), the main effect is an enormous variation of the mean height of the charged colloids by a factor $Z$, i.e., from the gravitational length $L \equiv k_{B} T / m g$ in the barometric (high-salt) regime to $Z L$ in the deionized case [17,21-24]. The explanation that was given in Ref. [17] invokes the salt ion entropy (which favors a homogeneous ion distribution) and the electrostatic energy (which favors local charge neutrality) to the total free energy, besides the gravitational energy and colloid entropy. The key physics is then that the total free energy is dominated, at low salinity, by the counterion entropy such that the system strives to obtain a rather homogeneous counterion distribution. Because of the tendency to satisfy local charge neutrality combined with the absence of coions (at low salinity), the colloids have to be distributed such that local neutrality is obtained, i.e., they have to be lifted upward toward the counterions. This is accomplished by a macroscopic charge separation at the system boundaries or at crossover heights (at the expense of electrostatic energy), such that an electric field is generated to lift the colloids upward against the gravitational pull [17]. We note here that it was recently shown that this lift effect can also be understood on the basis of effective colloid-colloid repulsions within a one-component view of the suspension combined with hydrostatic equilibrium $[25,26]$. 
The same lift mechanism for the colloids was shown to persist in the case of binary mixtures of charged colloids at low salinity, at least in simulations and in theory $[18,19,27]$; we are not aware of experiments in this direction. Besides the lifting effect, however, an additional segregation effect was found in these mixtures, whereby layers of essentially pure species $i$ are formed according to the product $Z_{i} L_{i}$, with $Z_{i}$ the charge number and $L_{i}$ the gravitational length of colloidal species $i$ : the average height of species $i$ was found to increase with charge per mass $Z_{i} L_{i}$ at low salinity (and with inverse mass at high salinity) $[18,19]$. In the case that $L_{1}<L_{2}$ (species 1 is heavier than 2) but with $Z_{1} L_{1}>Z_{2} L_{2}$ (species 1 is sufficiently more charged than 2) this leads to a layer with the heavier species 1 floating on top of a layer of the lighter species 2. This inversion was called the "colloidal Brazil nut effect" in Ref. [18], and the simulations of this effect presented in Ref. [18] could be described quantitatively by the inhomogeneous Poisson-Boltzmann theory of Ref. [19]. It was shown recently that this effect can also be obtained in computer simulations using an effective onecomponent description with effective pair potentials only [28]. Besides binary mixtures, also three-component and polydisperse mixtures of charged colloids were studied theoretically in Ref. [19]. For these systems the lifting effect at low salinity persisted, as well as the segregation into layers of identical charge per mass. So far, however, these predictions for multicomponent colloidal suspensions, which are based on a mean-field-like Poisson-Boltzmann theory, have not been tested against primitive model simulations. Given that this layering effect could be exploited to, e.g., purify a mixture of charged colloids, it is of practical importance to establish this effect, and to assess the accuracy of the theory and the regime of its breakdown. We expect the theory to break down at high enough packings and/or at strong enough electrostatic couplings. For these reasons we present in this paper the results of our Monte Carlo simulations of sedimentation equilibrium in multicomponent and monocomponent charged colloidal systems. These systems are described on the basis of the primitive model, i.e., the colloids and the counterions are taken into account explicitly, interacting through pairwise hard-core and Coulombic potentials, while the solvent is viewed as a dielectric continuum [29].

This paper is organized as follows. In Sec. II, we define the details of the model and describe some of the computational details. In Sec. III, we present the resulting equilibrium profiles and compare them with those predicted by the theory presented in Ref. [19]. Section IV is devoted to discussion and conclusions.

\section{MODEL AND SIMULATION METHOD}

We have performed Monte Carlo (MC) simulations at constant temperature and volume (MC NVT) of the primitive model of a deionized $n$-component colloidal system subject to a homogeneous external gravity field in the negative vertical $z$ direction. Every colloidal species $i$ (with $i=1, \ldots, n$ ) has the same hard-core diameter $\sigma_{i}=\sigma$, a fixed positive charge $Z_{i} e$ in its center (with $Z_{i}>0$ ), a buoyant mass $m_{i}$, and a gravitational length $L_{i}=k_{B} T / m_{i} g$. The number of colloidal particles of species $i$ is denoted by $N_{i}$. In addition, the system contains $N_{-}$monovalent negative counterions (charge $-e$ ) which are massless. Global charge neutrality dictates that $N_{-}=\sum_{i} N_{i} Z_{i}$, such that the total number of particles in the simulation is $N=\sum_{i} N_{i}+N_{-}$. The hard-core diameter of the ions is in all cases $\sigma_{-}=\sigma / 19$, i.e., much smaller than the colloidal diameter $\sigma$, but finite in order to prevent a collapse of the system. We also introduce the Bjerrum length $\lambda_{B}=e^{2} / \epsilon k_{B} T$, with $\epsilon$ the dielectric constant of the suspending medium. We set $\lambda_{B}=4 \times 10^{-3} \sigma$ throughout this study. The underlying microscopic Hamiltonian is therefore identical to the one in the theoretical study of Ref. [19], except perhaps for the finite size of the microscopic ions in the simulations compared to point ions in the theory. Given the small ionic diameter compared to the colloidal one (combined with the low ion concentrations of interest here), we do not expect any significant effect from this small difference.

The pair potential $U_{i j}\left(r_{i j}\right)$ between a particle of species $i$ and $j$ at separation $r_{i j}$ is given by

$$
\beta U_{i j}\left(r_{i j}\right)=\left\{\begin{array}{cc}
\infty, & r_{i j} \leqslant \sigma_{i j}, \\
\frac{Z_{i} Z_{j}}{r_{i j}} \lambda_{B}, & r_{i j} \geqslant \sigma_{i j},
\end{array}\right.
$$

where $i, j \in\{1, \ldots, n,-\}$ label either colloidal or ionic components, $\beta=1 / k_{B} T$ is the reciprocal temperature, $\sigma_{i j}=\left(\sigma_{i}+\sigma_{j}\right) / 2$ is the contact distance between the pair $i j$, and where we understand that $Z_{-}=-1$ for the counterions. In addition to the interaction potential between the particles the gravitational field acts on the colloids, as described by the external potential $\beta V_{i}(z)=\beta m_{i} g z=z / L_{i}$ for colloidal species $i$. This potential only affects the colloids, the microions are considered massless.

The simulation box that we used has dimensions $L_{x} \times L_{y} \times H$, such that the vertical $z$ coordinate is restricted to $z \in[0, H]$ with $H$ the height of the sample, while the horizontal in-plane coordinates are restricted to $x, y \in[0, K]$, with $K=L_{x}=L_{y}$. Periodic boundary conditions are only applied in the $x y$ directions, not in the $z$ direction. In all our simulations we set $H \simeq 100 \sigma$, and typically $K \ll H$, with $K \in[4.8 \sigma, 7.4 \sigma]$.

In order to speed up the simulations, we implemented the lattice method proposed by Panagiotopoulos and Kumar [30]. In this method, the particles are constrained to the discrete positions of a simple cubic lattice with lattice spacing $a$, such that all particle-particle interactions are calculated (and stored) only once at the beginning of the simulation and read out (without calculation) during the simulation. The lattice discretization is characterized by the parameter $\xi \equiv \sigma_{+-} / a$, where $\sigma_{+-}=\left(\sigma+\sigma_{-}\right) / 2$, and is such that $\xi \rightarrow \infty$ corresponds to the continuum limit of interest, whereas $\xi=1$ is the lattice gas model. In this work we set $\xi=10$, which we consider a good balance and which was also used previously by other authors for similar systems [22,31]. Moreover, it is known from earlier work that the difference between the critical parameters of electrolytes at $\xi=10$ and $\xi \rightarrow \infty$ is small, about $1 \%$ for the critical temperature and about $4 \%$ for the density [32]. 
The long-range character of the Coulomb interaction is such that a proper evaluation of the total Coulomb energy $U$ of the present system (no periodicity in the $z$ direction) must include the contributions from particles in all the periodic images in the $x$ and $y$ directions. An efficient way to accomplish this is the Ewald summation technique, which is a standard textbook method $[33,34]$, at least for bulk systems with periodic images in all three directions. However, for the socalled slab geometry [34-37] of interest here, the standard three-dimensional Ewald summation method (EWD3D) must be adjusted; see, e.g., [36] and references therein for several approaches to this problem. In the Appendix we compare two ways to implement the Ewald summation in a slab geometry, the exact two-dimensional Ewald summation (EWD2D), which is inconvenient because of the high computational cost, and the three-dimensional Ewald summation with an additional slab of vacuum and a correction for the spurious dipolar interactions (EWD3DC). Comparing both methods, we find that the EWD3DC method introduces an artifact when the system presents an intrinsic dipolar moment. In order to avoid the artifacts associated with the corrected EWD3D method we have chosen to implement the exact, but computationally expensive, EWD2D method [38-40] in this work. The computational cost is, however, significantly reduced by combining it with the lattice method, in which all interactions are precalculated only once and stored as discussed above.

For all simulations that are presented in this paper, we fix the height of the box to $H=100 \sigma$, with variations of the order of a few $\sigma$ such that the box size is compatible with the dimensions of the lattice. The horizontal dimensions $L_{x}$ and $L_{y}$ are chosen such that the packing fraction of the system matches with a required value, for a fixed number of particles. The parameters of the EWD2D method are the same as described in the Appendix. The colloidal charge numbers used in this work $(Z \in[6,100])$ are lower than in typical realistic colloidal suspensions. We have restricted ourselves to these values for practical reasons: the total number of particles in the system is otherwise too big to obtain equilibrated configurations in a reasonable simulation time, while the physical phenomena can still be revealed for lower colloid charges.

For a similar reason we present here only simulations for deionized systems. We have performed some tests with low salt densities, but we found similar behavior as in the deionized situation. In order to find stronger differences, it would be necessary to increase the salt concentration considerably, and thereby, the number of particles in the simulations and, hence the simulation time. Our simulations for low ionized systems show the same level of agreement with the theory of [19] than the deionized system presented in this paper.

A typical simulation consists of 50000 cycles to equilibrate the system and 100000 cycles to obtain the averages. A cycle consists of $N$ trials to move a randomly chosen colloid or microion (with $N$ the total number of counterions and colloids in the system). In a dense system of microions, a simple Monte Carlo move of a colloid would almost certainly result in a hard-core overlap with one of the microions. In order to avoid such overlaps we use a "cluster move" technique, where ions that overlap with the new colloid po-

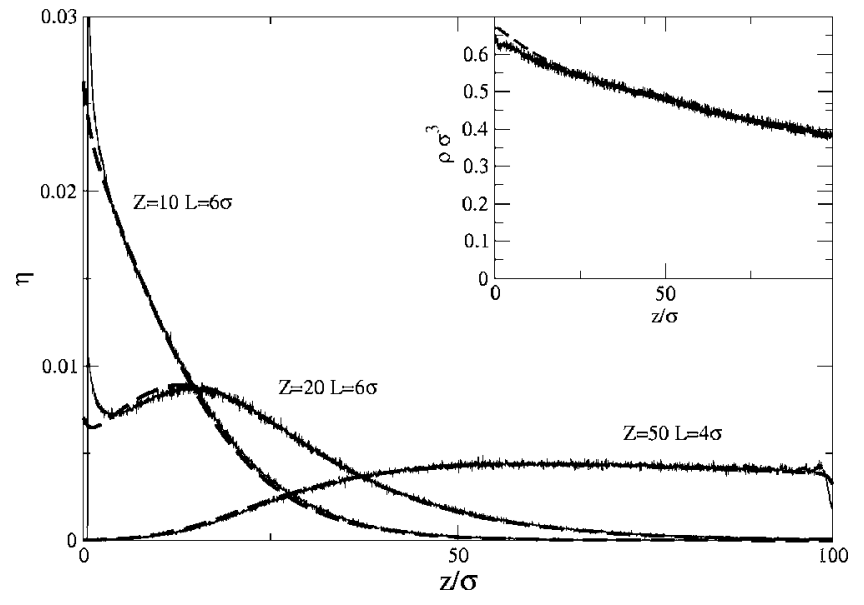

FIG. 1. Packing fraction profiles $\eta(z)$ for an equimolar ternary colloidal mixture with height $H=100 \sigma$ and $L_{x}=L_{y}=7.4 \sigma$. The total packing fraction is $\eta=0.0095$ and the colloids have charge and gravitational length $(Z, L / \sigma)=(50,4),(20,6)$, and $(10,6)$, respectively. The inset shows the density profile for the microions in the system. The dashed lines are the theoretical predictions of [19] and the solid lines the present simulation results.

sition are moved into the space left empty by the displaced colloid; more details on this technique can be found in Refs. $[31,41]$. The percentage of accepted moves of each component (colloids and microions) was maintained at $\approx 40 \%$. To check if the system is equilibrated, the center of mass of the colloids was monitored in the simulation. If this center of mass was not stable along the simulation, further equilibration took place before measurements were taken. All initial configurations of the simulations were random distributions of colloids and microions. The simulations are computationally more expensive with increasing microion densities. For instance, the simulation for $Z=100$ at a colloid packing fraction $\eta=0.0093$ takes about one month of AMD Athlon 64 $3200+$ processor time. We are therefore restricted to colloidal charges $Z \leqslant 100$ and low colloid densities.

\section{RESULTS AND DISCUSSION}

The first system that we consider represents a deionized ternary mixture $(n=3)$ with colloid charges and gravitational lengths given by $\left(Z_{i}, L_{i} / \sigma\right)=(50,4),(20,6)$, and $(10,6)$, for $i=1,2$, and 3 , respectively. The mixture is equimolar with a total packing fraction $\eta=0.0095$. These parameters are such that $Z_{i} L_{i} / \sigma=200,120$, and 60 for $i=1,2$, and 3. Hence one expects, on the basis of the theory of Ref. [19], a pronounced segregation into three layers with species 3 at the bottom (because it has the smallest value for $Z_{i} L_{i}$ ), species 1 at the top (because it has the highest value $Z_{i} L_{i}$ ), and species 2 in between. This is indeed what was found in our simulations (see Fig. 1), where the three packing fraction profiles are shown. The theoretical predictions, based on the theory of Ref. [19], are represented by the dashed curves, that agree perfectly with the simulations.

The second system we consider is deionized equimolar mixture with $n=5$ colloidal components with a total packing 


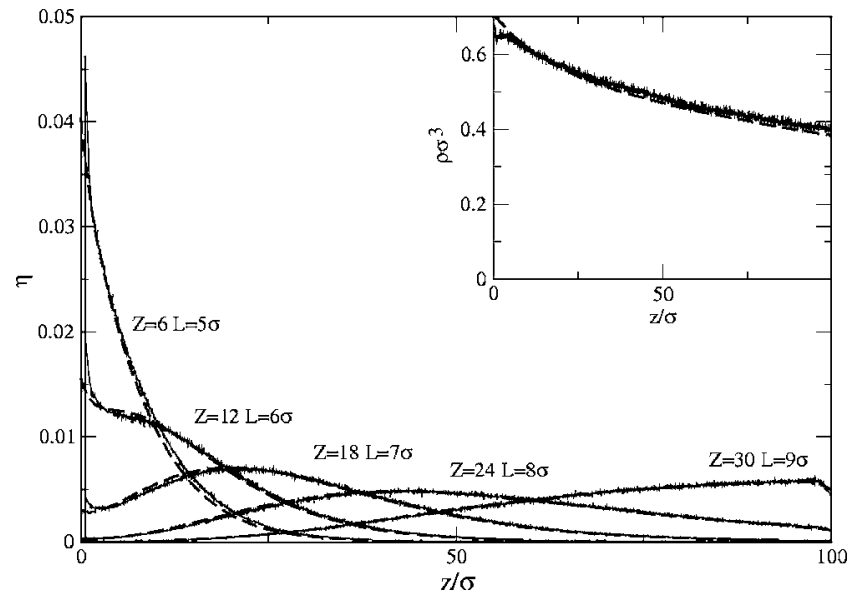

FIG. 2. Packing fraction profiles $\eta(z)$ for an equimolar fivecomponent colloidal mixture with height $H=100 \sigma, L_{x}=L_{y}=7.4 \sigma$, total packing fraction $\eta=0.014$, and colloids with charge and gravitational length $(Z, L / \sigma)=(6,5),(12,6),(18,7),(24,8)$, and $(30,9)$. The inset shows the density profile for the microions in the system. The dashed lines are the theoretical prediction of [19] and the solid lines the present simulation results.

fraction of $\eta=0.014$. The charges and the gravitational lengths are $\left(Z_{i}, L_{i} / \sigma\right)=(6,5),(12,6),(18,7),(24,8)$, and $(30,9)$, respectively. In Fig. 2 we show the packing fraction profiles of this system. In Figs. 1 and 2, the counterion profiles are shown in the inset; the counterions span the whole simulation box, in agreement with the notion that the ionic entropy is close to optimal in the low-salt regime studied here. The dashed curves are the theoretical predictions based on Ref. [19], which are again in quantitative agreement with the simulations. Also the ordering of the layers according to $Z_{i} L_{i}$ is as predicted in Refs. [18,19].

In order to further compare the simulation results with the theoretical predictions, we also measured the total charge density profile

$$
q(z)=-\rho_{-}(z)+\sum_{i=1}^{n} Z_{i} \rho_{i}(z)
$$

of the systems studied in Figs. 1 and 2. The result is shown in Fig. 3, and reveals for both cases a pronounced net positive charge close to the bottom and a net negative charge close to the top, combined with an essentially locally neutral regime in the "bulk" $(10 \lesssim z / \sigma \lesssim 90)$ of the system. This is in agreement with the theory represented by the dashed curves. The charge distributions satisfy global charge neutrality exactly, and give rise to an electric field similar to that of a plate condenser with two opposite surface charges on the parallel plates. The strength of this electric field $E(z)$ at height $z$ is related to the charge distribution $q(z)$ as follows from integrating the Poisson equation

$$
\frac{E(z) e \sigma}{k_{B} T}=4 \pi \frac{\lambda_{B}}{\sigma} \sigma^{2} \int_{0}^{z} q\left(z^{\prime}\right) d z^{\prime} .
$$

In Fig. 4, we plot this spontaneously formed electric field for the three- and five-component systems that we investigated,

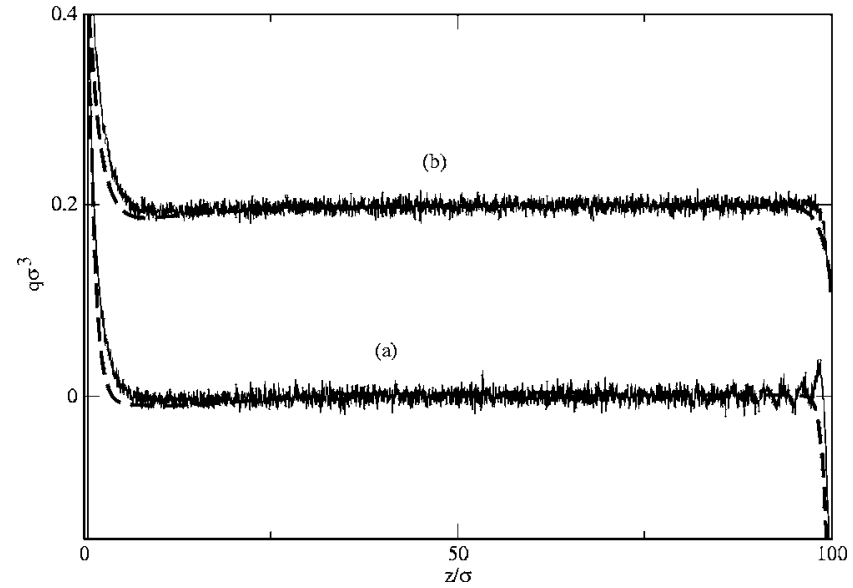

FIG. 3. Total charge density profile $q(z)$ [see Eq. (2)], obtained from simulation (solid line) and theoretically by Ref. [19] (dashed line). (a) and (b) correspond to the systems described in Figs. 1 and 2 , respectively. For clarity, the graph for (b) is shifted upward by 0.2 .

together with the theoretical predictions. The agreement is pretty good, although there are some deviations between theory and simulation, which are probably due to finite particle sizes and correlations that are ignored in the theory. We also see that the electric field is rather homogeneous in the bulk, but not exactly, indicating that the charge distribution is small but not exactly vanishing in the bulk.

The simulations presented so far correspond to systems with relatively low colloid charges and low total packing fractions. Our simulations provide a confirmation of the theoretical predictions on the segregation into layers of sedimenting multicomponent mixtures of charged colloids and they show that the accuracy of the theory [19] is of high level for the system parameters considered here. An immediate question that arises concerns the limitations of the theory. In order to investigate the break down of the theory, we perform simulations at higher total densities for monodis-

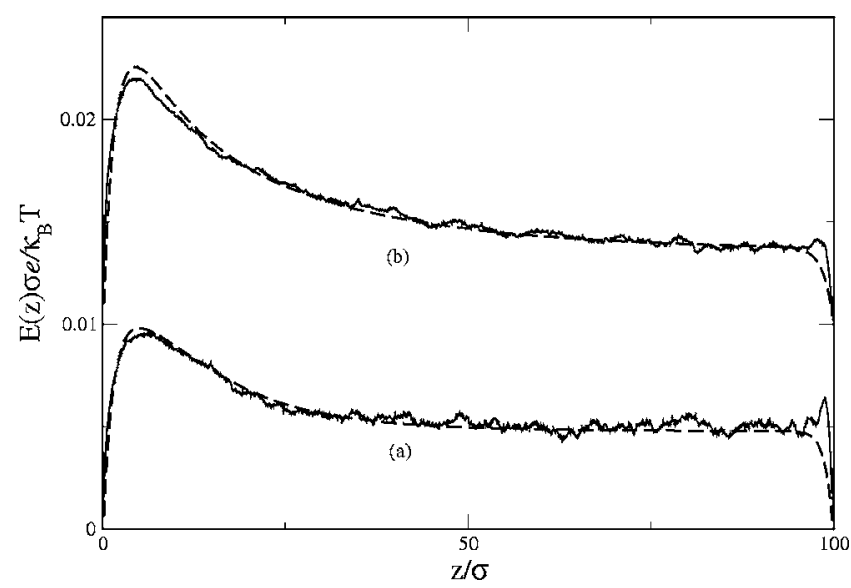

FIG. 4. Dimensionless electric field defined by Eq. (3) from the simulations (solid line) and predicted theoretically by [19] (dashed line). The profiles labeled (a) and (b) correspond to the systems described in Figs. 1 and 2, respectively. For clarity, the graph (b) is shifted upward by 0.01 . 


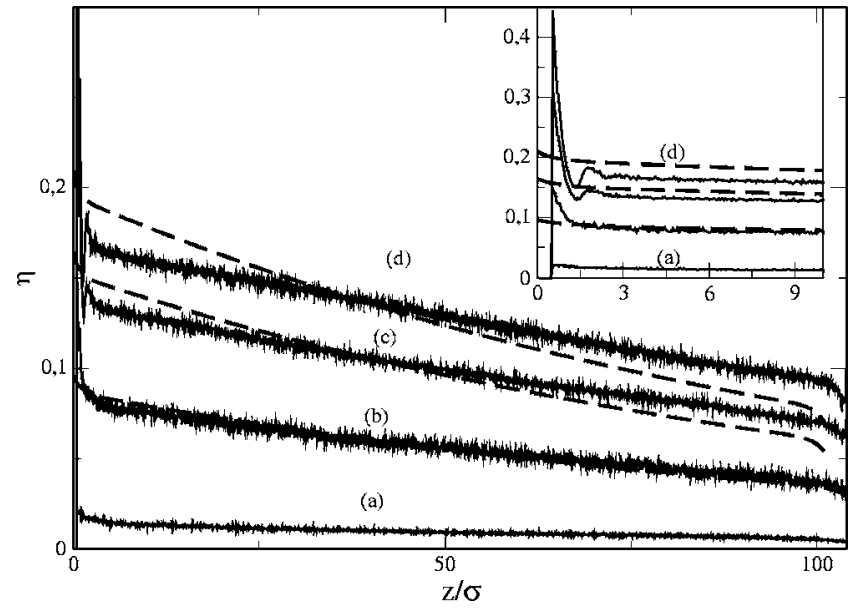

FIG. 5. Packing fraction profiles $\eta(z)$ for a monodisperse suspension of colloids with a charge $Z=10$, gravitational length $L / \sigma=10$, and height $H=103 \sigma$ and $L_{x}=L_{y}=4.8 \sigma$. The profiles labeled (a)-(d) correspond to total packing fractions $\eta=0.0093$, $0.055,0.099$, and 0.128 , respectively. The inset show the packing fraction profile near the bottom wall at an expanded scale. The dashed lines denote the theoretical predictions of [19] and the solid lines are the simulation results.

perse and multicomponent systems and at stronger electrostatic couplings by increasing the colloidal charge for monodisperse suspensions. The theory presented in Ref. [19] ignores the hard core of the colloidal particles and we therefore expect it to break down at higher packing fractions. In Fig. 5, we show the packing fraction profiles $\eta(z)$ for a monodisperse system of colloids with a gravitational length $L=10 \sigma$ and colloidal charge $Z=10$ and total colloidal packing fraction $\eta=0.0093,0.055,0.099$, and 0.128 , respectively. The packing fraction profile close to the bottom wall is shown at an expanded scale in the inset. For comparison, we present the theoretical predictions, based on the theory of Ref. [19], by the dashed curves. The most dilute system has been studied previously by Hynninen et al. using molecular dynamics simulations [31]. Figure 5 shows at this dilute packing fraction the same level of agreement between theory and simulation as reported previously [31]. In addition, Fig. 5 shows deviations between simulations and theory upon increasing the total packing fraction. Both the theory and the simulations show a linear behavior of the packing fraction profile, but the slope predicted by the theory is higher than that found by simulations. The more expanded packing fraction profiles found in the simulations is due to the hard-core interactions of the colloids that expel the colloidal particles from the bottom of the container to higher altitudes, where the density is lower. The hard-core interactions are, however, neglected in the theory, resulting in a more inhomogeneous packing fraction profile. In addition, the inset shows that the density of the first fluid layer increases enormously upon increasing $\eta$, while the "second" fluid layer is less pronounced. The formation of fluid layers at the wall due to packing effects is not predicted by theory, as the theory neglects the excluded volume interactions between the colloids and of the colloids with the wall.

In Fig. 6 we study the equimolar ternary mixture described in Fig. 1, but at a higher total packing fraction, i.e,

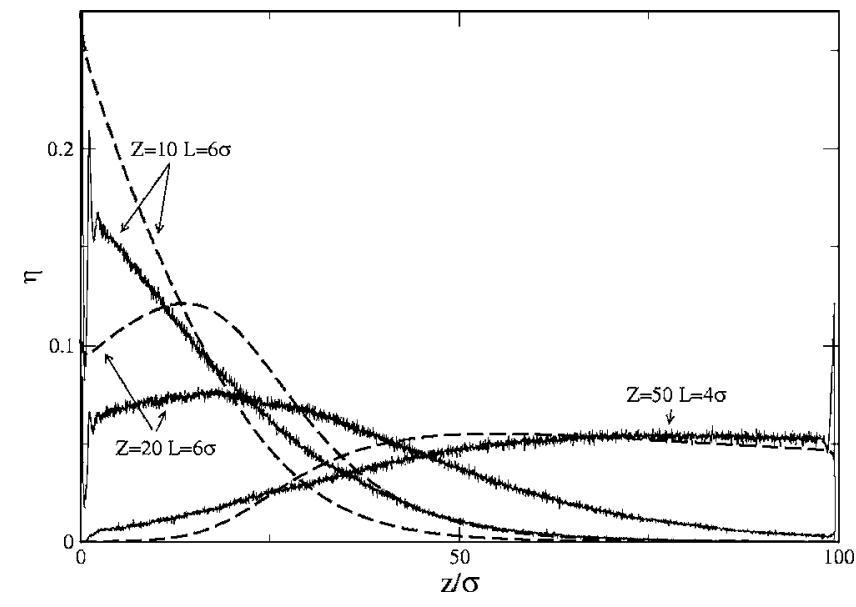

FIG. 6. Packing fraction profiles $\eta(z)$ for an equimolar ternary colloidal mixture with height $H=100 \sigma$ and $L_{x}=L_{y}=7.4 \sigma$. The colloids have identical charges and gravitational lengths as in Fig. 1. The total packing fraction is $\eta=0.115$. The dashed lines are the theoretical predictions of [19] and the solid lines the simulation results.

$\eta=0.115$. We again observe as in Fig. 1, a segregation of the components into three layers, where colloids with large charge-to-mass ratio sediment higher in the sample. The theoretical predictions agree qualitatively with the simulations, but quantitatively we do find deviations. As in the monodisperse case, we again find that the packing fraction profiles obtained from simulations are more expanded than those obtained from theory due to hard-core interactions. Moreover, we find at this high packing fraction the formation of fluid layers close to the bottom wall due to packing effects. These oscillations in the profiles are absent in the theoretical predictions for reasons discussed above.

We also studied the effect of electrostatic coupling by increasing the colloidal charge. In Fig. 7, we plot the packing fraction profiles $\eta(z)$ obtained from simulations (solid line) and theory (dashed line), for monodisperse colloidal suspensions with gravitational length $L=10 \sigma$ and colloidal charges $Z=0,10,20$, and 100 , respectively. The total packing fraction is $\eta=0.0093$. Figure 7 shows that the distribution of the colloids in the system becomes more homogeneous upon increasing the colloidal charge. In addition, Fig. 7 shows that the simulated packing profile differs from the theoretical prediction for $Z=0$. The theory predicts a barometric distribution, while the profile obtained from simulation is more expanded due to excluded volume effects. At $Z=100$, the packing fraction profile is completely flat in the bulk, apart from some structure close to the bottom and upper wall. The theoretical predictions are in good agreement with the simulation results for the colloidal charges studied here. Again we find a peak in the packing fraction profiles close to the walls. The peak moves away from the walls upon increasing the colloidal charge and as the effective diameter of the colloids increases.

Finally, we explore the effect of the composition of a colloidal binary mixture $(n=2)$ on segregation under gravity. To this end we studied a rather asymmetric deionized colloidal mixture with charges and gravitational lengths given 


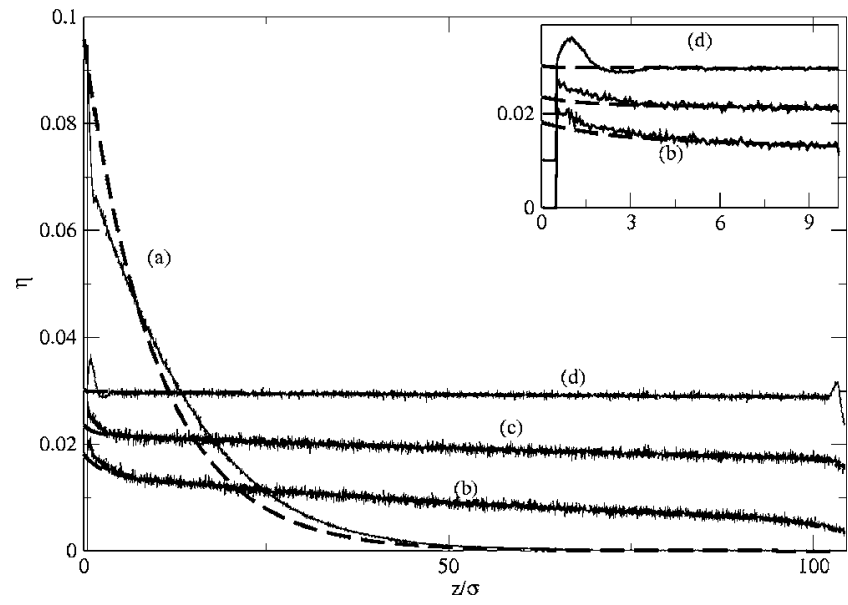

FIG. 7. Packing fraction profiles $\eta(z)$ for a monodisperse colloidal suspension with total packing fraction $\eta=0.0093$, height $H=103 \sigma, L_{x}=L_{y}=7.4 \sigma$, gravitational length $L=10 \sigma$, and colloidal charge $Z=$ (a) 0 , (b) 10 , (c) 20, and (d) 100. The inset shows the packing fraction profiles for $Z=10,20$, and 100 , near the bottom wall at an expanded scale. The dashed lines are the theoretical predictions of [19] and the solid lines simulation results. For clarity the graphs (c) and (d) are shifted upward by 0.01 and 0.02 , respectively.

$\left(Z_{i}, L_{i} / \sigma\right)=(50,1)$ and $(10,10)$ for $i=1$ and 2 , respectively. In Fig. 8, we show the simulated density profiles for (a) the equimolar case with $\eta_{1}=\eta_{2}=0.01$, and (b) the case where the lighter component is ten times more dilute such that $\eta_{1}=0.01$ and $\eta_{2}=0.001$. In both cases the layered structure persists, with the lighter component 2 floating on top of the heavier component 1 since $Z_{2} L_{2}>Z_{1} L_{1}$, in agreement with earlier conclusions $[18,19]$. An important difference, certainly in the light of a possible purification application, is the

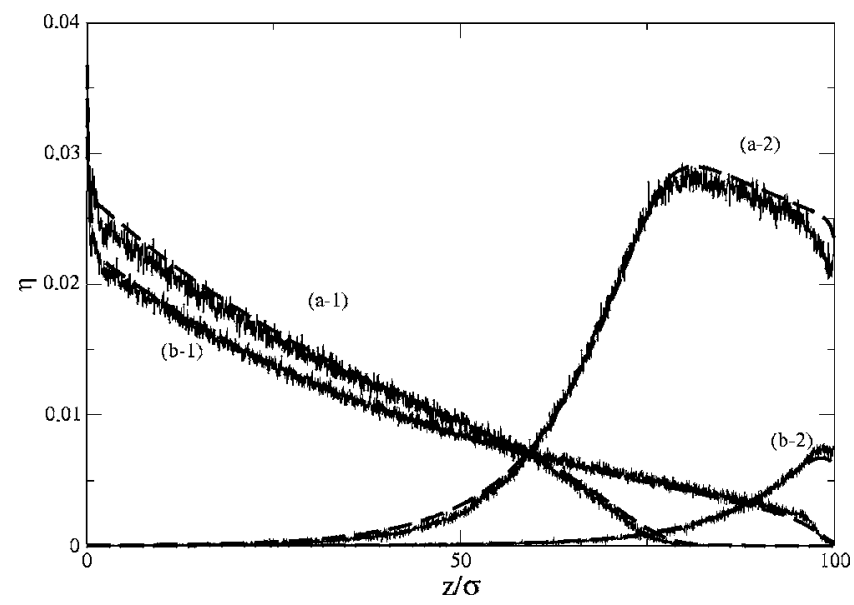

FIG. 8. Packing fraction profiles $\eta(z)$ for binary mixtures of colloids with charge and gravitational length $(Z, L / \sigma)=(50,1)$ and $(10,10)$. The (a) labeled profiles correspond to an equimolar mixture where each component has packing fraction $\eta_{i}=0.01$. The (b) labeled profiles correspond to a situation where the packing fraction of the heaviest component is $\eta=0.01$ and for the lighter $\eta=0.001$. (a-1) and (b-1) correspond in each situation to the heaviest component, while (a-2) and (b-2) correspond to the lighter component. The dashed lines are the theoretical predictions of [19] and the solid lines the present simulation results. complete expulsion of the heavier component 1 from the top $20 \sigma$ of the suspension below the meniscus in the equimolar case (a) compared to the considerable local mixing of the two species in this part of the sample in (b). The separation of the two components suggests that this phenomenology may be used to separate and extract components from a solution, even for trace components. The main difference between this case and the binary mixtures studied by Esztermann and Löwen [18] is the higher asymmetry between the charge and gravitational length of both species in the present case. In the situation presented here this asymmetry enhances the separation of the components. Figure 8 shows that the theoretical predictions agree perfectly with the simulations and, so the theory of Ref. [19] can be used to estimate the optimal parameters to separate the components.

\section{SUMMARY AND CONCLUSIONS}

We have performed MC simulations and PoissonBoltzmann calculations of sedimentation equilibrium of multi-component colloidal suspensions within the primitive model. Our simulations of sedimentation equilibrium of equimolar ternary and five-component systems are in quantitative agreement with the Poisson-Boltzmann theory of Ref. [19] in situations with low colloidal charge and dilute regimes, and thus confirm the existence of the lift effect of the colloids to much higher altitudes than expected from their buoyant mass as well as the separation according to mass per charge in multicomponent systems of charged colloids at low salinity. It may be of interest to explore the possibility to purify colloidal mixtures on this basis.

In addition, we have performed computer simulations in a regime where we expect the theory to break down, i.e., at high packing fractions and high electrostatic couplings by increasing the colloidal charge. We find deviations between simulations and theory upon increasing the total densities in the monodisperse and multicomponent systems. The density profiles obtained from simulations are more homogeneous than those predicted by theory. The more expanded profiles obtained from simulations are a direct consequence of the excluded volume interactions between the colloids, which are ignored in the theory. We do find good agreement between theory and simulation when the colloidal charge is increased in the monodisperse case for the parameters we investigated here.

So far, we have not been able to test all aspects of the theory. For instance, we have only considered here some deionized cases, whereas an interesting prediction concerns the crossover to ideal-gas-like (or hard-sphere-like) sedimentation at sufficiently high salt concentrations. This crossover salt concentration is, however, so high that the number of ions is exceedingly large.

\section{ACKNOWLEDGMENTS}

The lattice Monte Carlo code is based on A. Z. Panagiotopoulos' original code. This work is part of the research program of the "Stichting voor Fundamenteel Onderzoek der Materie," which is financially suported by the 
"Nederlandse Organisatie voor Wetenschappelijk Onderzoek (NWO)."

\section{APPENDIX: EWALD SUMMATION FOR A SLAB GEOMETRY}

Below we discuss two approaches to handle the longrange interaction in a slab geometry, the exact twodimensional Ewald summation and the three-dimensional Ewald summation with an additional slab of vacuum and a correction for the spurious dipolar interactions. Comparing both methods, we find that the EWD3DC method introduces an artifact when the system presents an intrinsic dipolar moment.

The two-dimensional Ewald summation (EWD2D) [38-40] is the most accurate approach, in which the total Coulombic energy of $N$ particles is given by

$$
\begin{aligned}
\frac{\beta U_{i j}}{\lambda_{B}}= & \frac{1}{2} \sum_{i, j=1}^{N} \sum_{|m|=0}^{\infty}{ }^{\prime} Z_{i} Z_{j} \frac{\operatorname{erfc}\left(\alpha\left|\mathbf{r}_{i j}+\mathbf{m}\right|\right)}{\left|\mathbf{r}_{i j}+\mathbf{m}\right|}+\frac{\pi}{2 A} \\
& \times \sum_{i, j=1}^{N} \sum_{\mathbf{h} \neq 0} Z_{i} Z_{j} \frac{\cos \left(\mathbf{r}_{i j} \cdot \mathbf{h}\right)}{h}\left\{\exp \left(h z_{i j}\right) \operatorname{erfc}\left(\alpha z_{i j}+\frac{h}{2 \alpha}\right)\right. \\
& \left.+\exp \left(-h z_{i j}\right) \operatorname{erfc}\left(-\alpha z_{i j}+\frac{h}{2 \alpha}\right)\right\} \\
& -\frac{\pi}{A} \sum_{i, j=1}^{N} Z_{i} Z_{j}\left\{z_{i j} \operatorname{erf}\left(\alpha z_{i j}\right)+\frac{1}{\alpha \sqrt{\pi}} \exp \left(-\alpha^{2} z_{i j}^{2}\right)\right\} \\
& -\left(\frac{\alpha}{\sqrt{\pi}}\right) \sum_{i=1}^{N} Z_{i}^{2}
\end{aligned}
$$

where $\mathbf{m}=\left(m_{x} L_{x}, m_{y} L_{y}, 0\right)$ is the lattice vector with $m_{x}, m_{y}$ integers and $L_{x}=L_{y}=K$ is the box dimension in the $x$ and $y$ directions. We also introduced the reciprocal lattice vector given by $\mathbf{h}=\left(2 \pi m_{x}^{\prime} / L_{x}, 2 \pi m_{y}^{\prime} / L_{y}, 0\right)$ with integer $m_{x}^{\prime}, m_{y}^{\prime}$, and the area of the unit cell $A=L_{x} L_{y}$. The primed sum indicates the omission of the $i=j$ term when $\mathbf{m}=\mathbf{0}, \alpha$ and the number of vectors $\mathbf{m}$ and $\mathbf{h}$ are adjustable parameters chosen such that computational efficiency is optimized. erf( ) and erfc( ) denote the error function and the complementary error function, respectively.

Even though Eq. (A1) is very accurate (provided the correct parameters are chosen), it is inconvenient to use in practice because of the high computational cost associated with the double sum over the particles in the Fourier part of the expression. For this reason several approximations have been proposed to speed up the computation of the long-range interactions in the slab geometry. One of these is to periodically repeat the cell in the $z$ direction such that the more efficient Ewald summation in three dimensions can be used, which then has to be corrected in order to remove the contribution from the repeating dipole (and higher order multipoles) in the $z$ direction (EWD3DC) [34-37]. The lowestorder correction term is given by [42]

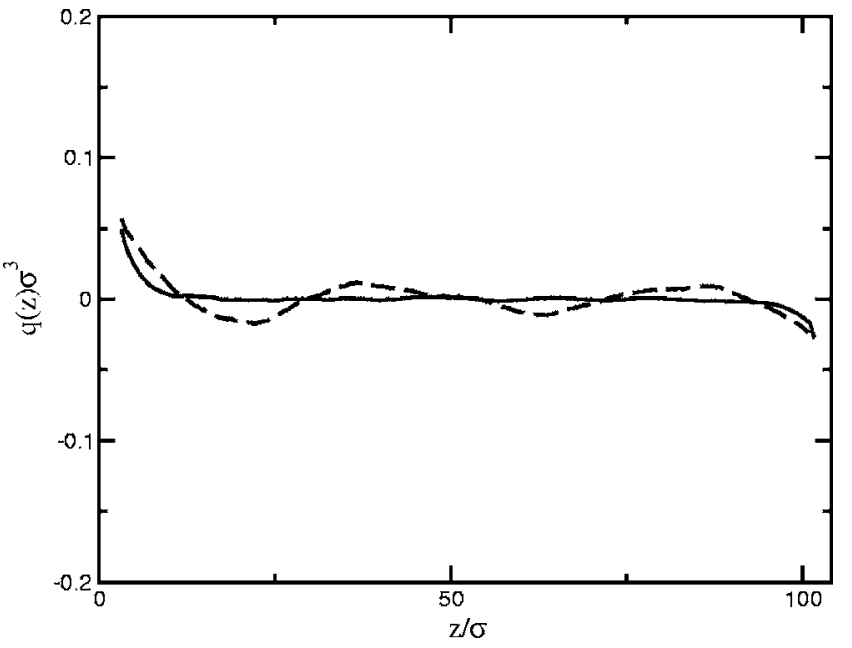

FIG. 9. Comparison between the charge profile obtained with the EWD3DC method (dashed line) and the EWD2D method (solid line). The profiles are coarse grained to facilitate the comparison. See the main text for details.

$$
\frac{\beta U_{c}}{\lambda_{B}}=\frac{2 \pi}{V}\left(\sum_{i}^{N} Z_{i} z_{i}\right)^{2}
$$

where $V$ is the volume of the box. It was shown in Ref. [36] that applying this correction to the EWD3D sum gives a result in excellent agreement with the more expensive EWD2D result given by Eq. (A1). However, our tests revealed a pronounced difference between the results of the more expensive EWD2D and the corrected EWD3DC method. The test result that we present in Fig. 9 is one of the cases studied by molecular dynamics simulations in Ref. [24], and represents the total charge distribution of a deionized monodisperse colloidal system $(n=1)$ with $L / \sigma=10$, $Z=10, \lambda_{B}=4 \times 10^{-3} \sigma, H=100 \sigma$, and total colloidal packing fraction $\eta=0.01$. Our Ewald summations were implemented with 112 Fourier-space vectors and $\alpha=0.68 \sigma^{-1}$, with the real-space sum in $U$ limited to the central cell. To facilitate comparison, we coarse-grain the density profiles in Fig. 9. The EWD2D result, which we find to be indistinguishable from the simulations and the theory of Ref. [24], reveals a charge separation such that the bottom $(z \approx 0)$ is positively charged while the meniscus $(z \approx H)$ is negatively charged. The resulting electric field pushes the (positive) colloids up against gravity. This result is quite different from the charge profile obtained with the EWD3DC method, which shows oscillations with a periodicity $\sim H / 2$ and an amplitude of the same magnitude as the charge density that causes the electric field with the EWD2D method. A tentative explanation for these oscillations, which we consider to be artefacts, is that the lowest-order correction of the EWD3DC method is most likely not sufficient in slab geometries with an intrinsic dipole moment. We note that this does not necessarily imply that the EWD3DC cannot be used at all in such cases, but it seems that one should correct for higher-order multipoles as well. In order to avoid the artifacts associated with the corrected EWD3D method we have chosen to implement the EWD2D method in this work. 
[1] J. Perrin, Les Atomes (Alcan, Paris, 1913).

[2] R. Piazza, T. Bellini, and V. Degiorgio, Phys. Rev. Lett. 71, 4267 (1993).

[3] M. A. Rutgers, J. H. Dunsmuir, J-Z. Xue, W. B. Russel, and P. M. Chaikin, Phys. Rev. B 53, 5043 (1996).

[4] T. Biben, J. P. Hansen, and J. L. Barrat, J. Chem. Phys. 98, 7330 (1993).

[5] T. Biben and J. P. Hansen, J. Phys.: Condens. Matter 6, A345 (1994).

[6] M. Dijkstra, J. P. Hansen, and P. A. Madden, Phys. Rev. Lett. 75, 2236 (1995).

[7] M. Dijkstra, J. P. Hansen, and P. A. Madden, Phys. Rev. E 55, 3044 (1997).

[8] S. V. Savenko and M. Dijkstra, Phys. Rev. E 70, 051401 (2004).

[9] T. Biben, R. Ohnesorge, and H. Löwen, Europhys. Lett. 28, 665 (1994).

[10] R. Allen, D. Goulding, and J.-P. Hansen, PhysChemComm 2, 30 (1999).

[11] V. A. Baulin and A. R. Khokhlov, Phys. Rev. E 60, 2973 (1999).

[12] D. van der Beek, T. Schilling, and H. N. W. Lekkerkerker, J. Chem. Phys. 121, 5423 (2004).

[13] H. H. Wensink and H. N. W. Lekkerkerker, Europhys. Lett. 66, 125 (2004).

[14] M. Schmidt, M. Dijkstra, and J. P. Hansen, Phys. Rev. Lett. 93, 088303 (2004).

[15] M. Schmidt, M. Dijkstra, and J.-P. Hansen, J. Phys.: Condens. Matter 16, S4185 (2004).

[16] T. Biben and J. P. Hansen, Mol. Phys. 80, 853 (1993).

[17] R. van Roij, J. Phys.: Condens. Matter 15, S3569 (2003).

[18] A. Esztermann and H. Löwen, Europhys. Lett. 68, 120 (2004).

[19] J. Zwanikken and R. van Roij, Europhys. Lett. 71, 480 (2005).

[20] A. P. Philipse and G. H. Koenderink, Adv. Colloid Interface Sci. 100-102, 613 (2003).

[21] M. Raşa and A. P. Philipse, Nature (London) 429, 857 (2004).
[22] C. P. Royall, R. van Roij, and A. van Blaaderen, J. Phys.: Condens. Matter 17, 2315 (2005).

[23] M. Rasa, B. Erné, B. Zoetekouw, R. van Roij, and A. P. Philipse, J. Phys.: Condens. Matter 17, 2293 (2005).

[24] A.-P. Hynninen, R. van Roij, and M. Dijkstra, Europhys. Lett. 65, 719 (2004).

[25] A. Torres and R. van Roij (unpublished).

[26] L. Belloni, J. Chem. Phys. 123, 204705 (2005).

[27] P. M. Biesheuvel and J. Lyklema, J. Phys.: Condens. Matter 17, 1 (2005).

[28] M. Dijkstra, J. Zwanniken, and R. van Roij, J. Phys.: Condens. Matter 18, 825 (2006).

[29] J. P. Hansen and I. R. McDonald, Theory of Simple Liquids (Academic Press, New York, 1990).

[30] A. Z. Panagiotopoulos and S. K. Kumar, Phys. Rev. Lett. 83, 2981 (1999).

[31] A.-P. Hynninen and M. Dijkstra, J. Chem. Phys. 123, 244902 (2005).

[32] A. Z. Panagiotopoulos, J. Chem. Phys. 116, 3007 (2002).

[33] M. P. Allen and D. J. Tildesley, Computer Simulation of Liquids (Clarendon Press, Oxford, 1987).

[34] D. Frenkel and B. Smit, Understanding Molecular Simulation (Academic Press, New York, 2002).

[35] I.-C. Yeh and M. L. Berkowitz, J. Chem. Phys. 111, 3155 (1999).

[36] P. S. Crozier, R. L. Rowley, E. Spohr, and D. Henderson, J. Chem. Phys. 112, 3155 (2000).

[37] A. Grzybowski, E. Gwóźdź, and A. Bródka, Phys. Rev. B 61, 6706 (2000).

[38] D. E. Parry, Surf. Sci. 55, 195 (1976).

[39] D. M. Heyes, M. Barber, and J. H. R. Clarke, J. Chem. Soc., Faraday Trans. 2 73, 1485 (1976).

[40] S. W. de Leeuw and J. W. Perram, Mol. Phys. 37, 1313 (1979).

[41] V. Lobaskin and K. Qamhieh, J. Phys. Chem. B 107, 8022 (2003).

[42] E. R. Smith, Proc. R. Soc. London, Ser. A 375, 475 (1981). 